\title{
Association of Plasma Homocysteine Level and Carotid Intima-Media Thickness in Rheumatoid Arthritis Patients Receiving Methotrexate
}

\author{
Emrullah HAYTA, ${ }^{1}$ Sami HİZMETLİ, ${ }^{1}$ Mehmet Haydar ATALAR, ${ }^{2}$ Ziynet ÇINAR ${ }^{3}$ \\ ${ }^{1}$ Department of Physical Medicine and Rehabilitation, Medical Faculty of Cumhuriyet University, Sivas, Turkey \\ ${ }^{2}$ Department of Radiology, Medical Faculty of Cumhuriyet University, Sivas, Turkey \\ ${ }^{3}$ Department of Biostatistics, Medical Faculty of Cumhuriyet University, Sivas, Turkey
}

\begin{abstract}
Objectives: This study aims to evaluate the relationship between serum homocysteine levels and atherosclerotic plaques in carotid arteries in female patients with rheumatoid arthritis (RA).

Patients and methods: Fifty-eight females with RA (mean age $45.50 \pm 11.69$ years; range 42 to 60 years) and 22 female controls (mean age $47.3 \pm 8.9$ years; range 38 to 62 years) with mechanical low back pain were included in the study. RA patients were divided into two groups: patients who were under methotrexate (MTX) therapy at least for two years (MTX group, $n=32$ ), and patients who did not receive MTX at least for past two years (nonMTX-disease-modifying antirheumatic drug group, $n=26$ ). RA patients' disease activity scores in 28 joints were calculated. Carotid intima-media thickness and presence of atherosclerotic plaque were evaluated by high-resolution B-mode ultrasonography.

Results: Plasma homocysteine levels were higher in MTX and non-MTX-disease-modifying antirheumatic drug groups compared to controls $(16.88 \pm 6.84$ and $10.37 \pm 2.54 \mu \mathrm{mol} / \mathrm{L}$, respectively) and the difference was statistically significant $(p=0.001)$. In MTX, non-MTX-disease-modifying antirheumatic drug and control groups, atherosclerotic plaque was detected in 11 (34.5\%) and four (15.4\%) patients, and one patient (4.5\%), respectively. Carotid intima-media thickness was significantly higher in MTX group compared to other two groups and the difference was statistically significant $(\mathrm{p}=0.002)$. Pearson correlation analysis revealed a significant correlation between intima-media thickness and homocysteine levels in MTX group $(r=0.49, p=0.006)$

Conclusion: According to our study results, methotrexate treatment in female patients with RA increases plasma homocysteine levels and prevalence of atherosclerotic plaque. High homocysteine levels in these patients may be a cause of atherosclerosis.

Keywords: Atherosclerosis; B-mode ultrasonography; homocysteine; rheumatoid arthritis.
\end{abstract}

Rheumatoid arthritis (RA) is a systemic and progressive disease with a chronic course and multifactorial origin. It is characterized with chronic inflammation and immune response in appropriate genetic background, and it causes functional loss and mortality in the long-term. And also, it is the most common autoimmune disease in inflammatory arthritic conditions. ${ }^{1}$

In patients with RA, life expectancy is 3-10 years shorter than normal population. ${ }^{2}$ Cardiovascular diseases (CVD) including vascular diseases of the brain are responsible of $40-50 \%$ of the deaths in RA. ${ }^{2,3}$ Intima-media thickness (IMT) and plaque frequency were increased in subclinical atherosclerosis studies, and this may be a sign for early-onset atherosclerosis in RA. ${ }^{4}$ But, traditional risk factors for atherosclerotic CVD cannot clearly explain the high frequency of coronary artery diseases in this group. Commonly known traditional risk factors can only explain 50\% of the coronary heart disease cases in general population. ${ }^{5}$ Some authors agreed on the fact that RA was an independent risk factor for coronary heart disease such as diabetes mellitus and hypertension. Recent studies also showed that high plasma level of homocysteine (Hcy) was an independent risk factor for CVD. ${ }^{6}$ 
Homocysteine is sulfur including essential amino acid, and it is an intermediate product in the metabolism of methionine. It can easily be oxidized into various disulfide forms with its free sulfhydryl (thiol) group. Hcy is produced during the metabolism of methionine in the diet. Cystathionine beta-synthase or thermolabile methylenetetrahydrofolate reductase enzymes, and vitamins like B12, B6 and folic acid are the cofactors in the metabolism of Hcy. ${ }^{7}$ A negative correlation between these vitamins and Hcy levels was shown. ${ }^{8}$

High levels of Hcy are directly toxic for endothelial cells. They oxidize low-density lipoproteins and have prothrombotic effects. High Hcy levels are also related to atherosclerosis, and RA patients treated with methotrexate (MTX) have higher Hcy levels. It is postulated that high Hcy levels caused by MTX treatment in patients with RA increases the cardiac morbidity and mortality. ${ }^{9,10}$ According to our knowledge, there is a need to evaluate the impact of high Hcy levels on atherosclerosis of carotid arteries in patients receiving MTX treatment. Thus, in this study, we aimed to evaluate the relationship between serum Hcy levels and atherosclerotic plaques in carotid arteries in female patients with RA.

\section{PATIENTS AND METHODS}

This study included 58 females (mean age $45.50 \pm 11.69$ years; range 42 to 60 years) with $\mathrm{RA}$ and 22 female controls (mean age 47.3 \pm 8.9 years; range 38 to 62 years) with mechanical low back pain. After the approval of Human Ethics Committee of Cumhuriyet University, written informed consents were obtained from the participants. RA was diagnosed according to the revised criteria of American College of Rheumatology in 1987. ${ }^{11}$ RA patients were divided into two groups: patients administered MTX therapy at least for two years (MTX group, $\mathrm{n}=32$ ), and patients who did not use MTX in past two years [non-MTX-diseasemodifying antirheumatic drug (DMARD) group, $\mathrm{n}=26]$. All participants were selected among female patients since Hcy levels are related with sex. Patients administered anti-epileptic drugs or statins, who were smokers, and with complications of atherosclerosis (cerebrovascular disease, myocardial infarction) were excluded. Clinical characteristics including menopausal status, presence of hypertension, presence of diabetes mellitus, family history, dietary habits, physical activity levels, tobacco and alcohol consumption, and socio-cultural properties such as education and household income were recorded for each patient. Afterwards, disease activity scores in 28 joints as well as age, family history of atherosclerosis, duration of disease, family history of hypertension, steroid dose, body mass index, fasting blood glucose, lowdensity lipoproteins, high-density lipoproteins, Hcy, rheumatoid factor, folic acid, vitamin B12, erythrocyte sedimentation rate, and $\mathrm{C}$-reactive protein were recorded.

The patients fasted overnight and were not given any medication before blood collection. For the measurement of Hcy, blood was drawn 72 hours after last MTX dose. Five milliliter of blood samples were collected from antecubital veins into tubes containing $4.5 \mathrm{~mL}$ of ethylenediaminetetraacetic acid. Plasma was separated by 10 minutes of centrifugation at $5000 \mathrm{rpm}$, and was transported to the laboratory at $2-8{ }^{\circ} \mathrm{C}$, and preserved in $-20{ }^{\circ} \mathrm{C}$ for $8-10$ weeks until the analysis. Plasma Hcy levels ( $\mu \mathrm{mol} / \mathrm{L})$ were analyzed with ELx-800 equipment by ELISA method and AxisNorway kits. Plasma levels of Hcy were split into two groups as normal $(5-15 \mu \mathrm{mol} / \mathrm{L})$ and high (>15 $\mathrm{mmol} / \mathrm{L}) .^{12}$ Rheumatoid factor, sedimentation, C-reactive protein, total cholesterol, high-density lipoprotein, low-density lipoprotein, triglyceride, and glucose levels were measured daily. Folic acid and vitamin B12 levels were also measured, as the Hcy is a sensitive indicator of vitamin B12 and folic acid deficiencies.

Intima-media thickness can be measured via high-resolution ultrasonography and is considered to be a reliable marker of early generalized atherosclerosis, while the presence of atherosclerotic plaques indicates more manifestations of atherosclerotic changes. ${ }^{13}$ Adult studies revealed a significant increase in the risk of CVD when carotid IMT exceeds $0.8 \mathrm{~mm}$. We assessed carotid IMT by high-resolution B-mode ultrasonography in all patients, and examined the presence of atherosclerotic plaque. All evaluations were performed by experienced radiologists by an ultrasonography device (General Electric Logic 9; Waukesha, WI, USA) equipped with a $10-\mathrm{MHz}$ 
linear probe. Interobserver and intraobserver reliabilities were determined as Kappa coefficient ranged from $0.67-0.87 .{ }^{14,15}$

For the assessment of carotid IMT, participants were examined supine with the head rotated $45^{\circ}$ towards left or right side. Imaging was done in the plane parallel to the neck with the jugular vein lying immediately above the common carotid artery. Images of the right common carotid artery were centered 10 to $15 \mathrm{~mm}$ below (caudad to) the right common carotid artery bulb. End-diastolic images (smallest diameter of the artery) were captured. IMT of the common carotid artery and presence of plaques were assessed. IMT of the common carotid artery was determined in the far wall according $1 \mathrm{~cm}$ proximal to the bifurcation. Carotid artery plaque was excluded. ${ }^{16,17}$

\section{Statistical analysis}

The power of our study was 0.80462 when $\alpha, \beta$ and $1-\beta$ were considered as $0.05,0.20$ and 0.8 , respectively. SPSS for Windows version 14.0 (SPSS Inc., Chicago, IL, USA) software program was used for statistical analyses. Normality was tested with the Kolmogorov-Smirnov test. Comparison of the clinical and ultrasonographic data of the study groups were performed with Chi-square and ANOVA tests. Correlation of Hcy level and IMT were analyzed with Pearson correlation test. A $p$ value of less than 0.05 was accepted as significant.

\section{RESULTS}

Methotrexate doses ranged from 7.5 to $15 \mathrm{mg} /$ week in the MTX group. All patients taking MTX were also taking folic acid supplementation (10 mg/week). Table 1 presents the clinical and laboratory variables of MTX, non-MTX-DMARD, and control groups. There was no significant difference among the study groups with regard to the socio-demographic characteristics such as menopausal status, presence of hypertension, presence of diabetes mellitus, family history, physical activity, tobacco and alcohol consumption, education, and household income ( $p>0.05)$. The study groups were similar with regard to the parameters presented in Table 1 ( $p>0.05)$.

Figures 1 and 2 present the Hcy level and IMT value of the study groups. The Hcy level and IMT value of MTX group were significantly higher than those of the non-MTX-DMARD and control groups $(p<0.05)$. The non-MTX-DMARD and control groups were similar with regard to the Hcy level and IMT value ( $p>0.05)$. The Hcy levels and IMT values of non-MTX-DMARD and control groups were comparable ( $p>0.05)$.

Table 1. Characteristics of patients in rheumatoid arthritis and control groups

\begin{tabular}{|c|c|c|c|c|c|c|c|c|c|c|}
\hline & \multicolumn{3}{|c|}{$\begin{array}{l}\text { MTX group } \\
(\mathrm{n}=32)\end{array}$} & \multicolumn{3}{|c|}{$\begin{array}{l}\text { MTX-DMARD group } \\
(\mathrm{n}=26)\end{array}$} & \multicolumn{3}{|c|}{$\begin{array}{l}\text { Control group } \\
(\mathrm{n}=22)\end{array}$} & \multirow{2}{*}{$\begin{array}{c}\text { Significance } \\
p\end{array}$} \\
\hline & $\mathrm{n}$ & $\%$ & Mean \pm SD & $\mathrm{n}$ & $\%$ & Mean \pm SD & $\mathrm{n}$ & $\%$ & Mean \pm SD & \\
\hline Age (years) & & & $45.5 \pm 11.7$ & & & $47.9 \pm 10.1$ & & & $47.3 \pm 8.9$ & 0.66 \\
\hline Family history of atherosclerosis & 5 & 15.6 & & 4 & 15.4 & & 3 & 13.6 & & 0.97 \\
\hline Duration of rheumatoid arthritis & & & $8.9 \pm 8$ & & & $8.7 \pm 6$ & & & & 0.84 \\
\hline Presence of hypertension & 8 & 25 & & 6 & 23 & & 5 & 22.7 & & 0.97 \\
\hline Presence diabetes mellitus & 6 & 18 & & 7 & 27 & & 3 & 13.6 & & 0.50 \\
\hline Disease activity score 28 & & & $5.4 \pm 0.7$ & & & $5.3 \pm 0.7$ & & & & 0.91 \\
\hline Erythrocyte sedimentation rate & & & $46 \pm 5.6$ & & & $41 \pm 4.9$ & & & & 0.93 \\
\hline C-reactive protein & & & $6.4 \pm 1.9$ & & & $5.3 \pm 1.4$ & & & & 0.68 \\
\hline Rheumatoid factor positivity & 20 & 62.5 & & 19 & 73.1 & & & & & 0.39 \\
\hline Steroid dose & & & $6.7 \pm 2.9$ & & & $6.4 \pm 26$ & & & & 0.76 \\
\hline Body mass index $\left(\mathrm{kg} / \mathrm{m}^{2}\right)$ & & & $26.1 \pm 3.9$ & & & $26.2 \pm 3.5$ & & & $27.1 \pm 2.1$ & 0.50 \\
\hline Fasting blood glucose (mg/dL) & & & $96.5 \pm 27.3$ & & & $95.2 \pm 13.0$ & & & $93.2 \pm 13.1$ & 0.53 \\
\hline Total cholesterol (mg/dL) & & & $184.0 \pm 36.6$ & & & $179.2 \pm 38.4$ & & & $189.1 \pm 11.6$ & 0.53 \\
\hline Low-density lipoprotein (mg/dL) & & & $89.7 \pm 16.1$ & & & $89.2 \pm 13.5$ & & & $85.3 \pm 9.7$ & 0.49 \\
\hline High-density lipoprotein (mg/dL) & & & $38.6 \pm 10.1$ & & & $36.3 \pm 10.3$ & & & $35.1 \pm 4.7$ & 0.56 \\
\hline Folic acid $(\mathrm{pg} / \mathrm{mL})$ & & & $5.2 \pm 2.5$ & & & $6.5 \pm 3.1$ & & & $7.7 \pm 2.8$ & 0.86 \\
\hline Vitamin B12 (pg/mL) & & & $456 \pm 43.8$ & & & $385 \pm 64.1$ & & & $413 \pm 51.5$ & 0.65 \\
\hline
\end{tabular}




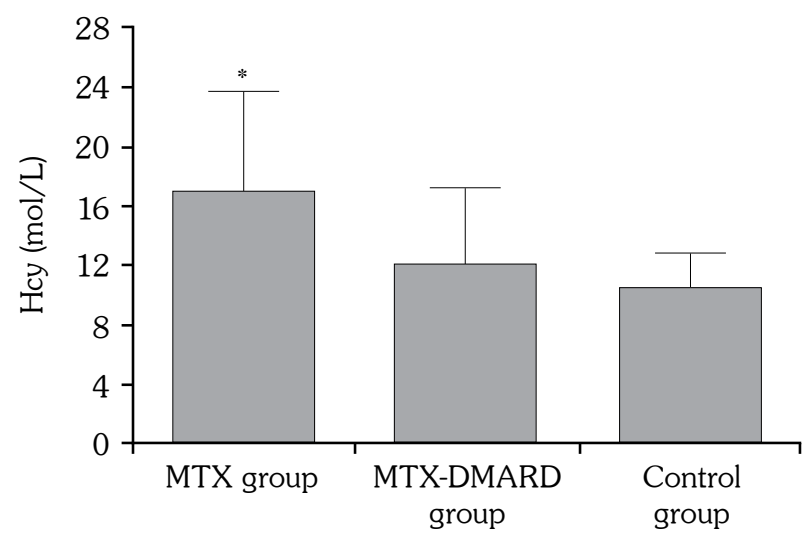

Figure 1. Homocysteine levels of study groups. ${ }^{*} p<0.05$ vs methotrexate (MTX)-disease-modifying antirheumatic drug (DMARD) and control groups; Hcy: Homocysteine.

Carotid plaque positivity was detected in $11(34.5 \%)$ and four (15.4\%) patients, and one (4.5\%) patient in the MTX, non-MTX-DMARD, and control groups, respectively (Figure 3). The ratio of carotid plaque positivity was significantly higher in the MTX group compared to the other groups $(p<0.05)$. Pearson correlation analysis revealed a significant correlation between IMT value and Hcy level only in the MTX group $(\mathrm{r}=0.49, \mathrm{p}=0.006)$.

\section{DISCUSSION}

We have shown that serum Hcy level and carotid IMT increased in patients with RA. The presence of atherosclerotic plaque was $25.86 \%$ in patients with RA and this shows the

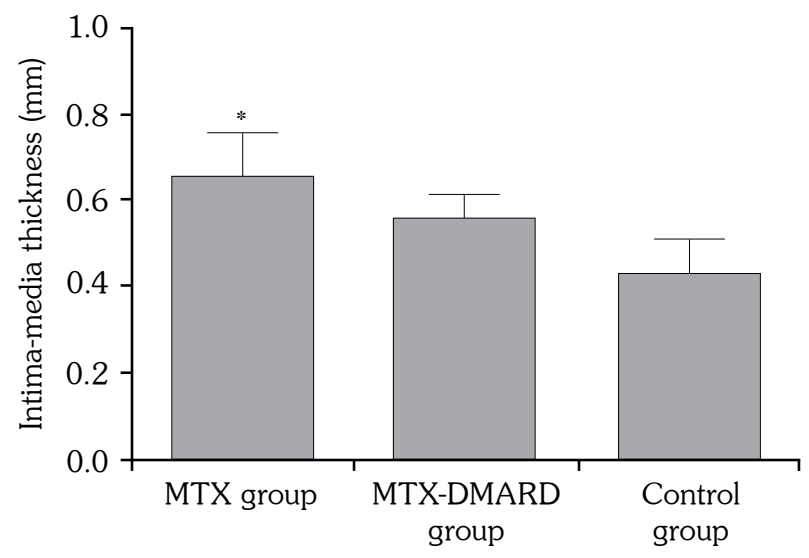

Figure 2. Intima-media thickness values of study groups. * p<0.05 vs methotrexate (MTX)-disease-modifying antirheumatic drug (DMARD) and control groups; Hcy: Homocysteine. accelerated atherosclerotic process and early plaque formation. The serum Hcy level and carotid IMT have a considerably significant correlation in patients with RA, particularly in those receiving MTX. Overall, these findings suggest that serum Hcy level and carotid IMT value provide important information about the status of large arteries (common carotid artery) related to the atherosclerotic process even before the clinical progression of cardiovascular risk/damage in RA. In addition, the Hcy level along with the ultrasonographic imaging of carotid arteries might be used for determining and following the severity of the vascular damage due to the inflammatory process of RA. ${ }^{18-20}$

Traditional risk factors for CVD alone cannot explain the increased mortality risk in RA. ${ }^{21}$ In recent studies, high homocysteine levels have been associated with an increased risk of cardiovascular events in the general population, and a 25\% lower homocysteine level was associated with an $11 \%$ lower risk of coronary artery disease and a 19\% lower risk of stroke. ${ }^{22,23}$ It was reported that every $5 \mathrm{mmol} / \mathrm{L}$ increase in Hcy increased the CVD risk 1.35 times in males, and 1.42 times in females. ${ }^{24}$ Other studies reported that hyperhomocysteinemia was an independent risk factor also for vascular thrombosis. ${ }^{25,26}$ A previous study in 2006 compared the Hcy levels between normal population and patients with RA, and suggested that Hcy levels were elevated in patients with RA, and that homocysteinemia must be treated. ${ }^{27}$ Likewise, our study revealed

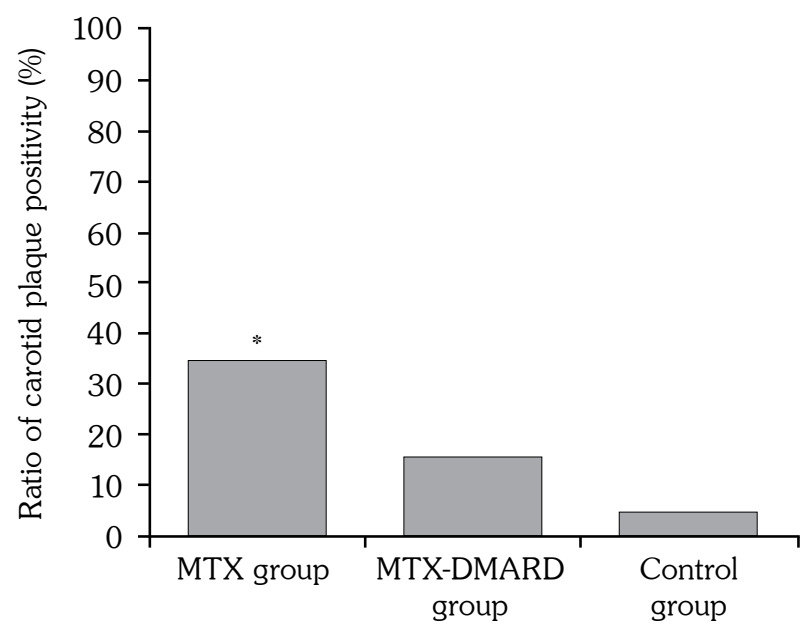

Figure 3. Ratio of carotid plaque positivity. ${ }^{*} p<0.05$ vs methotrexate (MTX)-disease-modifying antirheumatic drug (DMARD) and control groups. 
that patients with RA had higher Hcy levels when compared to controls.

Effects of MTX on Hcy levels in patients with RA are conflicting. Hoekstra et al. ${ }^{28}$ demonstrated that plasma levels of Hcy is elevated significantly after application of MTX, and gradually decreased to basal levels until the next application in patients with RA who are using high dose MTX (>25 mg/week). This weekly increase in Hcy levels after MTX treatment was postulated to increase the exposure to Hcy, and caused increased risk of CVD. Moreover, this study proposes that folic acid supplementation in patients with RA is ineffective for preventing against intermittent increases in Hcy. In spite of this study, Morgan et al. ${ }^{29}$ found that folic acid supplementation in patients receiving long-term low-dose MTX prevented folic acid deficiency, decreased hyperhomocysteinemia, and might provide a prevention against CVD progression in patients with RA. MTX treatment increases plasma levels of Hcy indirectly in patients with RA, and they must be supplemented with folic acid. ${ }^{30}$ Mean doses of $10 \mathrm{mg} /$ week of folic acid may not be enough and must be increased in patients with RA. While Haagsma et al. ${ }^{31}$ declared that MTX elevated the Hcy levels, Hernanz et al. ${ }^{32}$ have reported against this view. These conflicting reports may be explained by the timings of MTX treatment and obtaining blood samples for analyses. As we know that early folic acid supplementation in 48 hours can decrease the high Hcy levels, it should be rational to evaluate Hcy levels within first 48 hours in patients with RA.

Another method for assessing the increased risk of CVD and atherosclerosis in patients with $\mathrm{RA}$ is high-resolution B-mode ultrasonography. Carotid IMT was shown to be determinative when compared to controls in studies that evaluate the atherosclerosis by B-mode ultrasonography in patients with RA. ${ }^{33,34}$

Our study had some limitations such as relatively low number of patients, including only one sex, and short follow-up time. The complications, such as myocardial infarction and angina pectoris, due to the CVD in our patients were managed by our Cardiology Department, and these complications may be the focus of an another study.

In conclusion, according to our findings, the Hcy level increases in patients with RA, and this increase is more pronounced in patients receiving MTX. In patients with RA, particularly those administered MTX, the increase in carotid IMT and the presence of carotid plaque formation need to be considered. The meaningful association of Hcy level and IMT value is also important for physicians for the management and follow-up of the cases. Further studies are needed to elucidate the potential role of combined use of serum Hcy level and carotid IMT value and plaque formation as a test in RA patients who have an increased CVD risk.

\section{Declaration of conflicting interests}

The authors declared no conflicts of interest with respect to the authorship and/or publication of this article.

\section{Funding}

The authors received no financial support for the research and/or authorship of this article.

\section{REFERENCES}

1. Maini RN, Zvaifler NJ. Rheumatoid arthritis and spondyloarthropathy. In: Klippel JH, Dieppe PA, editors. Rheumatology. St Louis: Mosby; 1994. p. 3:1,1-40:1.

2. Kerekes G, Szekanecz Z, Dér H, Sándor Z, Lakos G, Muszbek L, et al. Endothelial dysfunction and atherosclerosis in rheumatoid arthritis: a multiparametric analysis using imaging techniques and laboratory markers of inflammation and autoimmunity. J Rheumatol 2008;35:398-406.

3. Kapetanovic MC, Lindquist E, Simonsson M, Geborek $\mathrm{P}$, Saxne T, Eberhardt K. Prevalence and predictive factors of comorbidity in rheumatoid arthritis patients monitored prospectively from disease onset up to 20 years: lack of association between inflammation and cardiovascular disease. Scand J Rheumatol 2010;39:353-9.

4. Ciftci O, Yilmaz S, Topcu S, Caliskan M, Gullu H, Erdogan D, et al. Impaired coronary microvascular function and increased intima-media thickness in rheumatoid arthritis. Atherosclerosis 2008;198:332-7.

5. del Rincón ID, Williams K, Stern MP, Freeman GL, Escalante A. High incidence of cardiovascular events in a rheumatoid arthritis cohort not explained by traditional cardiac risk factors. Arthritis Rheum 2001;44:2737-45.

6. van Ede $\mathrm{AE}$, Laan RF, Blom HJ, Boers $\mathrm{GH}$, Haagsma CJ, Thomas CM, et al. Homocysteine and folate status in methotrexate-treated patients with rheumatoid arthritis. Rheumatology (Oxford) 2002;41:658-65. 
7. Blom HJ, Smulders Y. Overview of homocysteine and folate metabolism. With special references to cardiovascular disease and neural tube defects. $\mathrm{J}$ Inherit Metab Dis 2011;34:75-81.

8. Klee GG. Cobalamin and folate evaluation: measurement of methylmalonic acid and homocysteine vs vitamin $\mathrm{B}(12)$ and folate. Clin Chem 2000;46:1277-83.

9. Ferretti G, Bacchetti T, Moroni C, Vignini A, Nanetti L, Curatola G. Effect of homocysteinylation of low density lipoproteins on lipid peroxidation of human endothelial cells. J Cell Biochem 2004;92:351-60.

10. Wållberg-Jonsson S, Ohman M, Rantapää-Dahlqvist $\mathrm{S}$. Which factors are related to the presence of atherosclerosis in rheumatoid arthritis? Scand J Rheumatol 2004;33:373-9.

11. Jacobsson LT, Knowler WC, Pillemer S, Hanson RL, Pettitt DJ, McCance DR, et al. A cross-sectional and longitudinal comparison of the Rome criteria for active rheumatoid arthritis (equivalent to the American College of Rheumatology 1958 criteria) and the American College of Rheumatology 1987 criteria for rheumatoid arthritis. Arthritis Rheum 1994;37:1479-86.

12. Kang SS, Wong PW, Malinow MR. Hyperhomocyst(e) inemia as a risk factor for occlusive vascular disease. Annu Rev Nutr 1992;12:279-98.

13. Jadhav UM, Kadam NN. Apolipoproteins: correlation with carotid intimamedia thickness and coronary artery disease. J Assoc Physicians India 2004;52:370-5.

14. Mitu F, Mitu M, Turiceanu M, Caliap TA, Leatu CG. The importance of carotid ultrasound in the risk stratification of patients with ischaemic heart disease. Rev Med Chir Soc Med Nat Iasi 2008;112:337-42. [Abstract]

15. Rosvall M, Janzon L, Berglund G, Engström G, Hedblad B. Incidence of stroke is related to carotid IMT even in the absence of plaque. Atherosclerosis 2005;179:325-31.

16. Polak JF, Backlund JY, Cleary PA, Harrington AP, O'Leary DH, Lachin JM, et al. Progression of carotid artery intima-media thickness during 12 years in the Diabetes Control and Complications Trial/Epidemiology of Diabetes Interventions and Complications (DCCT/EDIC) study. Diabetes 2011;60:607-13.

17. Kim HS, Cho KI. Association of carotid artery parameters of atherosclerosis in coronary artery disease. J Cardiovasc Ultrasound 2013;21:72-80.

18. Gómez-Vaquero C, Corrales A, Zacarías A, Rueda-Gotor J, Blanco R, González-Juanatey C, et al. SCORE and REGICOR function charts underestimate the cardiovascular risk in Spanish patients with rheumatoid arthritis. Arthritis Res Ther 2013;15:91.

19. Gonzalez A, Maradit Kremers H, Crowson CS, Ballman KV, Roger VL, Jacobsen SJ, et al. Do cardiovascular risk factors confer the same risk for cardiovascular outcomes in rheumatoid arthritis patients as in non-rheumatoid arthritis patients? Ann Rheum Dis 2008;67:64-9.

20. González-Gay MA, González-Juanatey C. Implication of new atherosclerotic carotid plaques in the cardiovascular outcome of patients with rheumatoid arthritis. Arthritis Res Ther 2012;14:105.

21. Gkaliagkousi E, Gavriilaki E, Doumas M, Petidis K, Aslanidis S, Stella D. Cardiovascular risk in rheumatoid arthritis: pathogenesis, diagnosis, and management. J Clin Rheumatol 2012;18:422-30.

22. Wald DS, Law M, Morris JK. Homocysteine and cardiovascular disease: evidence on causality from a meta-analysis. BMJ 2002;325:1202.

23. Homocysteine Studies Collaboration. Homocysteine and risk of ischemic heart disease and stroke: a metaanalysis. JAMA 2002;288:2015-22.

24. Cleophas TJ, Hornstra N, van Hoogstraten B, van der Meulen J. Homocysteine, a risk factor for coronary artery disease or not? A meta-analysis. Am J Cardiol 2000;86:1005-9.

25. Roubenoff R, Dellaripa P, Nadeau MR, Abad LW, Muldoon BA, Selhub J, et al. Abnormal homocysteine metabolism in rheumatoid arthritis. Arthritis Rheum 1997;40:718-22.

26. Cisternas M, Gutiérrez MA, Klaassen J, Acosta AM, Jacobelli S. Cardiovascular risk factors in Chilean patients with rheumatoid arthritis. J Rheumatol 2002;29:1619-22.

27. Lopez-Olivo MA, Gonzalez-Lopez L, GarciaGonzalez A, Villa-Manzano AI, Cota-Sanchez AR, Salazar-Paramo M, et al. Factors associated with hyperhomocysteinaemia in Mexican patients with rheumatoid arthritis. Scand J Rheumatol 2006;35:112-6.

28. Hoekstra M, Haagsma CJ, Doelman CJ, van de Laar MA. Intermittent rises in plasma homocysteine in patients with rheumatoid arthritis treated with higher dose methotrexate. Ann Rheum Dis 2005;64:141-3.

29. Morgan SL, Baggott JE, Lee JY, Alarcón GS. Folic acid supplementation prevents deficient blood folate levels and hyperhomocysteinemia during longterm, low dose methotrexate therapy for rheumatoid arthritis: implications for cardiovascular disease prevention. $\mathrm{J}$ Rheumatol 1998;25:441-6.

30. Yang HT, Lee M, Hong KS, Ovbiagele B, Saver JL. Efficacy of folic acid supplementation in cardiovascular disease prevention: an updated meta-analysis of randomized controlled trials. Eur J Intern Med 2012;23:745-54.

31. Haagsma CJ, Blom HJ, van Riel PL, van't Hof MA, Giesendorf BA, van Oppenraaij-Emmerzaal D, et al. Influence of sulphasalazine, methotrexate, and the combination of both on plasma homocysteine concentrations in patients with rheumatoid arthritis. Ann Rheum Dis 1999;58:79-84.

32. Hernanz A, Plaza A, Martín-Mola E, De Miguel E. Increased plasma levels of homocysteine and other 
thiol compounds in rheumatoid arthritis women. Clin Biochem 1999;32:65-70.

33. Cuomo G, Di Micco P, Niglio A, La Montagna G, Valentini G. Atherosclerosis and rheumatoid arthritis: relationships between intima-media thickness of the common carotid arteries and disease activity and disability. Reumatismo 2004;56:242-6. [Abstract]

34. Nagata-Sakurai $M$, Inaba $M$, Goto $H$, Kumeda $Y$, Furumitsu $\mathrm{Y}$, Inui $\mathrm{K}$, et al. Inflammation and bone resorption as independent factors of accelerated arterial wall thickening in patients with rheumatoid arthritis. Arthritis Rheum 2003;48:3061-7. 